\title{
SIMPLY CYCLIC HOMOGENEOUS NON-TREE-LIKE CURVES DECOMPOSE TO SOLENOIDS
}

JAMES T. ROGERS, JR.

(Communicated by Dennis Burke)

\begin{abstract}
It is proved that if a one-dimensional, cyclic, homogeneous continuum $X$ is the inverse limit of graphs each of which contains only one cycle, then $X$ is a solenoid or $X$ admits a decomposition into mutually homeomorphic, homogeneous, tree-like continua with quotient space a solenoid.
\end{abstract}

\section{INTRODUCTION}

A continuum is a compact, connected, nonvoid metric space. A curve is a one-dimensional continuum.

Each continuum can be represented as an inverse limit of compact polyhedra, and so each curve can be represented as an inverse limit of finite graphs. These representations are not unique.

A natural way to study curves is to classify them according to the graphs used in their inverse limit representations. A curve is said to be arc-like if it is an inverse limit of arcs, circle-like if it is an inverse limit of circles, and tree-like if it is an inverse limit of trees. Other such classifications are obvious. We assume the bonding maps of an inverse sequence are surjective.

An equivalent definition for a continuum to be arc-like is that it admits finite open covers of arbitrarily small mesh whose nerves are arcs. The words chainable and snake-like are often used to describe this definition. Circle-like and tree-like continua may also be defined in a similar fashion.

A curve $X$ is homogeneous if, for each pair of points $x$ and $y$ of $X$, there exists a homeomorphism of $(X, x)$ onto $(X, y)$. A solenoid is an inverse limit of circles with all the bonding maps being covering maps. Under this definition, the circle is a solenoid. Each solenoid is homogeneous, since it is a topological group.

Received by the editors January 30, 1989.

1980 Mathematics Subject Classification (1985 Revision). Primary 54F20; Secondary 54F50.

Key words and phrases. Continuum, curve, homogeneous, terminal subcontinuum, decomposition, simply cyclic.

This research was partially supported by a grant from the National Science Foundation. 
In 1959, Bing [1] began the program of classifying homogeneous curves by their representations via graphs by showing that the pseudo-arc is the only homogeneous, arc-like continuum. Interestingly, the pseudo-arc can also be represented as an inverse limit of circles, in which case most of the bonding maps must be inessential.

The second step was not taken until 1969. Following the independent results of Fearnley and of the author that the pseudo-circle is not homogeneous, Burgess [2] and Jones [5] independently showed that the homogeneous, circle-like, planar continua that are not arc-like are either circles or circles of pseudo-arcs, i.e., curves that admit a decomposition into pseudo-arcs with quotient space a circle.

Later, Hagopian and Rogers [4] extended this classification to all circle-like continua by showing that nonplanar, circle-like, homogeneous continua are either solenoids or admit a decomposition into pseudo-arcs with quotient space a solenoid.

The fourth step was taken recently by Lewis [6], who showed that if a homogeneous, tree-like curve admits a bound on the number of branching points of the trees in a defining inverse sequence, then it is a pseudo-arc. Whether or not a homogeneous, tree-like continuum must be a pseudo-arc is an important unsolved problem.

A curve is called simply cyclic if it is an inverse limit of graphs each of which contains only one cycle. Hence simply cyclic curves include circle-like curves as a proper subset. The purpose of this note is to take the next step in this program.

Theorem. If a simply cyclic, homogeneous curve is not tree-like, then either it is a solenoid or it admits a decomposition into mutually homeomorphic, tree-like, homogeneous curves with quotient space a solenoid.

In particular, this yields a new proof of the Hagopian-Rogers result classifying homogeneous, circle-like continua.

Our tools are the results in [7]. By restricting our attention to simply cyclic curves, we can avoid the use of hyperbolic geometry and deal with a Euclidean situation.

\section{COVERING SPACES OF SIMPLY CYCLIC CURVES}

Let $X$ be a homogeneous curve that is not tree-like. The author proved in [8] that such a curve must have nontrivial first Čech cohomology, i.e., $H^{1}(X) \neq 0$. It follows that there is an essential map $f: X \rightarrow S^{1}$. If $g: X \rightarrow Q$ is an embedding of $X$ into the Hilbert cube $Q$, then the map of $X$ into $S^{1} \times Q$ sending $x$ to $(f(x), g(x))$ is an essential embedding (i.e., the inclusion map is not homotopic to a constant map). Henceforth we consider $X$ to be essentially embedded into $S^{1} \times Q$. Let $p: R \times Q \rightarrow S^{1} \times Q$ be the universal covering space of $S^{1} \times Q$, where $p$ is the product of the exponential map on $R$ and the identity map on $Q$. The map $p$ is a local isometry. 
Let $K$ be a component of $p^{-1}(X)$. This construction is the Euclidean version of the hyperbolic construction of [8]; thus it follows from [8, Theorem 4.7] that the component $K$ is unbounded in both the positive and the negative direction. Hence $K$ has a natural two-point compactification $Y$.

A subcontinuum $Z$ of the continuum $X$ is said to be terminal if each subcontinuum $Y$ of $X$ that intersects $Z$ satisfies either $Y \subset Z$ or $Z \subset Y$. A decomposition of $X$ into continua is said to be terminal if each element of the decomposition is a terminal subcontinuum of $X$.

According to the main theorem of [7], the collection of maximal, terminal, proper subcontinua of $X$ exists and determines a continuous decomposition of $X$ into mutually homeomorphic, tree-like, homogeneous curves. Furthermore, the quotient space $X^{\prime}$ is a homogeneous curve that contains no proper, nondegenerate, terminal continuum, and $H^{1}\left(X^{\prime}\right) \neq 0$.

Finally, the collection of maximal, terminal, proper subcontinua of $X$ is generated by the collection of images of the maximal, terminal, proper subcontinua of $K$ under the covering map $p$.

Extend this decomposition of $X$ to a decomposition of $S^{1} \times Q$ by adding points as the other decomposition elements. Let $A$ be the quotient space, and let $f: S^{1} \times Q \rightarrow A$ be the quotient map. Since the nondegeneracy set $\left\{a \in A: f^{-1}(a)\right.$ is nondegenerate $\}$ is one-dimensional, it follows from results of Kozlowski [3, p. 115] that $A$ is an ANR. By Edwards' Theorem [3, p. 103], $f \times$ id: $S^{1} \times Q \times Q \rightarrow A \times Q$ is a near homeomorphism. Denote this near homeomorphism by $g: S^{1} \times Q \rightarrow S^{1} \times Q$. Note that $X^{\prime}$ is essentially embedded in $S^{1} \times Q$.

Since $X^{\prime}$ is a homogeneous curve essentially embedded in $S^{1} \times Q$, the results of [7] may be applied again, and we obtain a component $K^{\prime}$ of the preimage $p^{-1}\left(X^{\prime}\right)$. Furthermore, $K^{\prime}$ also has a natural two-point compactification $Y^{\prime}$.

The near homeomorphism $g: S^{1} \times Q \rightarrow S^{1} \times Q$ lifts to a map $\tilde{g}: R \times Q \rightarrow$ $R \times Q$. We assume that $\tilde{g}(K)=K^{\prime}$. Extend $\tilde{g}$ to a map (also called) $\tilde{g}: Y \rightarrow Y^{\prime}$ by mapping the two compactifying points of $Y$ onto the two compactifying points of $Y^{\prime}$. It follows that $Y^{\prime}$ is the quotient space of $Y$ under a cell-like decomposition.

Suppose that $X$ is simply cyclic. It follows that the curve $Y$ is tree-like. To see this, consider a simply cyclic cover of $X$ with mesh less than some positive number $\varepsilon$, and lift it to an open cover $\mathscr{C}$ of $K$ in $R \times Q$. Since $X$ is essentially embedded in $S^{1} \times Q$, the nerve of this open cover of $K$ is an infinite tree. Since $p$ is a local isometry, the mesh of this infinite cover is less than $\varepsilon$. The compactification $Y$ of $K$ has a metric yielding the topology on $Y$ such that the distance between the points $k_{1}$ and $k_{2}$ of $K$ in $Y$ is less than the distance between $k_{1}$ and $k_{2}$ in $R \times Q$. The continuum $Y$ is covered by an $\varepsilon$-tree chain consisting of a certain (finite) tree chain chosen from $\mathscr{C}$ and two small open sets containing the two compactifying points and the rest of $\mathscr{C}$. 
Since $Y^{\prime}$ is the quotient space of a cell-like decomposition of the tree-like continuum $Y$, it follows that $Y^{\prime}$ is also tree-like. Since $X^{\prime}$ satisfies the hypotheses of [7, Theorem 5.5], we conclude that either $X^{\prime}$ is a solenoid or $Y^{\prime}$ is a colocally connected curve, i.e., points have arbitrarily small neighborhoods with connected complements. A tree-like curve is not colocally connected. Hence $X^{\prime}$ is a solenoid. This completes the proof.

Corollary. Each circle-like continuum that is not a pseudo-arc is a solenoid or admits a decomposition into pseudo-arcs with quotient space a solenoid.

Proof. Each circle-like continuum is simply cyclic, each tree-like subcontinuum of a circle-like continuum is arc-like, and each homogeneous, arc-like continuum is a pseudo-arc.

\section{REFERENCES}

1. R. H. Bing, Each homogeneous nondegenerate chainable continua is a pseudo-arc, Proc. Amer. Math. Soc. 10 (1959), 345-346.

2. C. E. Burgess, A characterization of homogeneous plane continua that are circularly chainable, Bull. Amer. Math. Soc. 75 (1969), 1354-1356.

3. T. A. Chapman, Lectures on Hilbert cube manifolds, CBMS Regional Conf. Ser. in Math. No. 28, Amer. Math. Soc., Providence, RI (1976).

4. C. L. Hagopian and J. T. Rogers, Jr., A classification of homogeneous circle-like continua, Houston J. Math. 3 (1977), 471-474.

5. F. B. Jones, Homogeneous plane continua, Proc. Auburn Topology Conference (1969), 45-56.

6. I. W. Lewis, Homogeneous tree-like continua, Proc. Amer. Math. Soc. 82 (1981), 470-472.

7. J. T. Rogers, Jr., Decompositions of continua over the hyperbolic plane, Trans. Amer. Math. Soc. 308 (1988), 277-291.

8. _ Hyperbolic ends and continua, Michigan J. Math. 34 (1987), 337-347.

Department of Mathematics, Tulane University, New Orleans, Louisiana 70118 